\title{
Optical Phantom Development for Skin Measurement
}

\author{
Jing ZHANG ${ }^{1 *}$, Yuanjie LIU $^{1}$, Julie ROBIC ${ }^{2}$, Alex NKENGNE², Hong YAN ${ }^{3}$, Xing ZHANG ${ }^{3}$, Xiang Yun $\mathrm{SOO}^{3}$ \\ ${ }^{1}$ National Metrology Centre, Agency for Science, Technology and Research (A*STAR), Singapore \\ ${ }^{2}$ Clarins Laboratories, France \\ ${ }^{3}$ Institute of Materials Research and Engineering, A*STAR, Singapore \\ *Corresponding e-mail address: zhang_jing@nmc.a-star.edu.sg
}

\begin{abstract}
We have successfully developed and manufactured a three-layered scattering agent-embedded optical phantom in order to mimic the structural and optical properties of the human skin. Characterization is done for the optical phantom. The widths of the individual layers can be controlled within $+/-10 \%$ of the designed thickness. The refractive indices, scattering and absorption coefficients are close to the human skin. The scattering agents in the optical phantom act as point sources which can be used to measure point spread function (PSF). Such a device can be useful for applications that require an accurate optical model for human skin evaluation such as Optical Coherence Tomography (OCT) and Reflectance Confocal Microscopy (RCM) in cosmetics and dermatological diagnostics.
\end{abstract}

\section{Introduction}

OCT and RCM are 3D imaging technologies which can be used in cosmetic and medical applications. One common application in cosmetic and dermatology is the in vivo characterization of the skin morphological organization [1,2].

In order to develop confocal imaging systems, optical phantom are used either to develop, optimize or test the optical pathways, or to fine tune the image processing algorithms. These optical phantoms should mimics the optical properties of the skin, which means having three different layers with different optical refractive indexes. The phantoms also need to have microstructures that could mimic the refractive index changes in each layer related to the presence of cells and fibers. However, most of the optical phantoms are homogeneous without any microstructure to simulate the presence of cells and fibers [3]. Some optical phantoms only mimic the refractive index of one layer of the skin. Other phantoms mimic the scattering / absorption coefficients. Designing the optical phantom that is able to simulate the skin multi-layer structure and its optical property at the same time is a challenge. Our objective is to develop a multi-layer optical phantom that will demonstrate optical properties that are comparable to the skin. Developing such a phantom will allow to evaluate the performance of confocal imaging systems for in vivo skin imaging and to improve image processing of data acquired from those systems.

Early liquid phantoms suffer from short useful lives which are no more than two months, especially when scattering agents are embedded. As a result, recent efforts focus on producing long-lasting solid phantoms [4]. Solid phantoms based on optical-grade polymers, absorbers, and scattering agents offer long-term stability and reproducible optical properties, and are cost-effective referencing solutions. Representative prior inventions include polystyrene particles in polyvinyl alcohol (PVA) matrix [5] and polyvinyl chloride (PVC) layers bound with binary plasticizer 6].

In recent years, UV-curable resins have received increasing amount of attention due to their easy application, fast curing, excellent post-curing properties and absence of any volatile organic chemicals (VOC). Therefore, we aimed to exploit such features by making optical phantoms via successive layering of commercially available transparent UV-curable resins of appropriate thicknesses and refractive indices. Micrometer-scale glass beads were selected as scattering agents because they are chemically inert and have well-documented refractive indices and scattering coefficients.

\section{The structure of the skin}

The skin is composed of multiple layers (Fig. 1.a). The stratum corneum is the outer layer of the skin and consists of dead cells called corneocytes. Their cytoplasm shows filamentous keratin. The stratum cornum appears on confocal 
images as an amorphous bright structure with a high refractive index (Fig. 1.b). The living epidermis is located beneath the stratum corneum and contains epidermal cells, called keratinocytes, at different state of differentiation. The contrast on confocal images of the viable epidermis come from the difference in term of refractive index between the cytoplasm and the membrans of keratinocytes (Fig. 1.c). The stratum cornum and the viable epidermis provide a physical and chemical barrier against the environment. Beneath the epidermis, the dermis contains collagen and elastin fibers and tough connective tissue. The fibers appears on confocal images as contrasted fibrous structures which shape will change as a function of pathological or physiological states. Only the superficial epidermis can be imaged using the confocal in vivo microscopy since the classical depth for image acquisition will be around $\mathbf{2 5 0}$ microns. The hypodermis is the deeper layer of the skin and is made of fat and connective tissue. This layer is not reachable with the state-of-art in vivo microscopes.

The stratum corneum has a thickness between 10 and $40 \mu \mathrm{m}$. The epidermis is usually about $0.1 \mathrm{~mm}$ thick and can range from 0.07 to $0.12 \mathrm{~mm}$ or up to 0.8 to $1.4 \mathrm{~mm}$ for thicker part of the skin found in the palms and soles and the dermis thickness ranges from 0.3 to $4.0 \mathrm{~mm}$ [7].

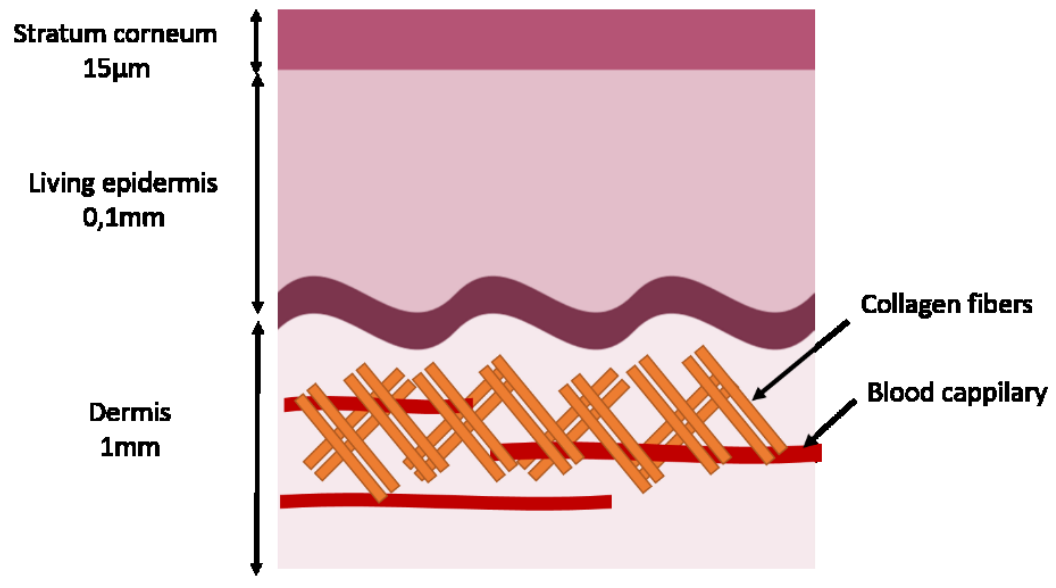

(a)

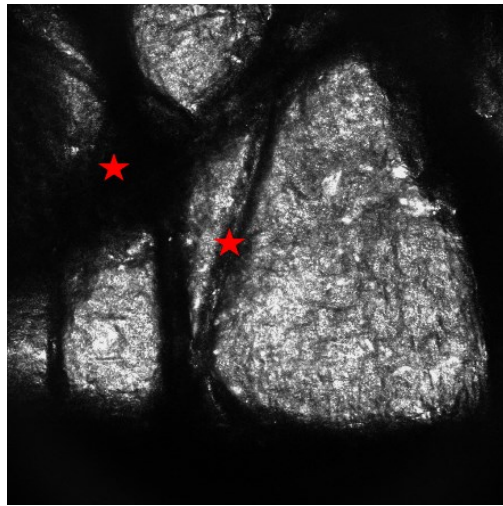

(b)

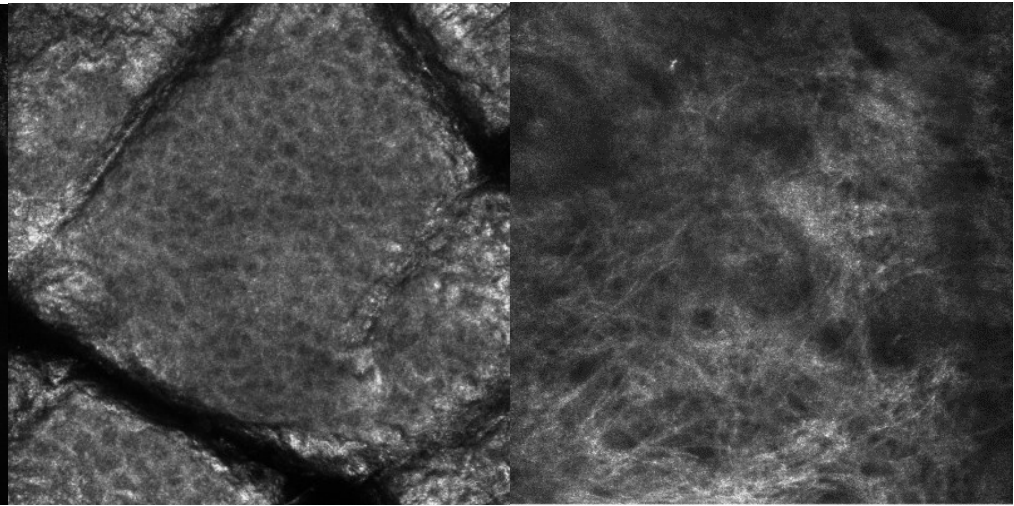

(d)

Fig. 1 Skin structure. (a) The different layers of the skin. Reflectance confocal image of the stratum cornum (b) , of the viable epidermis $(c)$ and the dermis (d)

\section{Optical property of the skin}

Normal skin has irregular air tissue interface, in which the refractive index changes from that of air, 1.0, to that of the skin. This causes a regular reflectance across the UV, visible, and near-infrared spectra. Ding et al. illuminated the skin in vitro using a number of light sources ranging in wavelength from 325 to $1557 \mathrm{~nm}$. They predicted values of refractive index ranging from approximately 1.41 to 1.49 in epidermal tissues and 1.36 to 1.41 in dermal tissues 
over the wavelength range [8]. By fitting the refractive index obtained at 8 wavelengths, they established the dispersion scheme of the in vitro dermis and epidermis in wavelength from 325 to $1557 \mathrm{~nm}$. However, Tearney et al. measured refractive indices of in vivo skin at $1300 \mathrm{~nm}$ and determined values from a single participant of 1.52 for the stratum corneum, 1.34 for the living epidermis and 1.41 for the dermis, suggesting that in vitro methods may result in an increase in the measured refractive indices [9].

Skin tissues are composed of organic matter and fits properly into the general class of dielectrics exhibit "body color" and weak, selective absorption. The epidermis depends on minor baseline skin absorption and dominant melanin absorption due to the melanosomes in the epidermis while the dermis depends on the hemoglobin absorption of the cutaneous blood. The absorption is proportional to the volume fractions of melanosomes and whole blood respectively.

The scattering of light in the dermis is dependent on the collagen content as it is optically birefringent and makes up around $70 \%$ of the dry weight of the dermis. As for the epidermal scattering, it is affected by the particulate scatter within the skin called filamentous proteins or keratin fibers. Since the scattering coefficient of the epidermis is close to that of the dermis and its thickness is adequately thin, dermal scattering is used instead to describe skin scattering when discussing devices and techniques which depend on photon diffusion. Tom Lister, et al. summarized the in vitro measurement results of optical scattering coefficient and absorption coefficient of human skin in [10]. The optical scattering coefficient of epidermis and dermis is in the range from $1 \mathrm{~mm}^{-1}$ to $10 \mathrm{~mm}^{-1}$, and the absorption coefficient is in the range from $0.1 \mathrm{~mm}^{-1}$ to $2 \mathrm{~mm}^{-1}$, respectively, in the wavelength range from $400 \mathrm{~nm}$ to $750 \mathrm{~nm}$.

\section{Design of the optical phantom}

The optical phantom is designed to mimic the multi-layer structure and optical properties (optical refractive index, optical scattering and absorption coefficients) of human skin from the epidermis to the dermis layer. Our design is for the reference for in vivo skin measurement at wavelength from $600 \mathrm{~nm}$ to $1300 \mathrm{~nm}$. Based on the skin layer structure from [7], in vivo skin refractive index from [9] and the dispersion scheme from [8], and optical scattering and absorption coefficient from [10], we design the optical phantom to have the parameters for each layer as shown in Fig. 2. The top layer has a refractive index close to 1.52-1.54 with thickness around 15 um, which is to mimic the stratum corneum. The middle layer has a refractive index close to 1.34-1.36 with thickness around 100 um in order to mimic the living epidermis. The bottom layer has a refractive index near 1.41-1.43 with thickness about $1 \mathrm{~mm}$ to mimic the dermis.

We use transparent epoxy as the materials for each layer. Commercially available transparent UV-curable resins with refractive indices in the range of the skin are used to make the layers of the optical phantom. The materials for the layers are selected to match the refractive index of the corresponding layer of the skin. Transparent material has very small scattering and absorption coefficients. To mimic the scattering and absorption effect in the skin, micrometer-scale glass beads are mixed in the layer as scattering agents to adjust the scattering and absorption coefficients. The diameters of the glass beads spread from $20 \mathrm{um}$ to $70 \mathrm{um}$. The glass beads have refractive index of 1.51-1.52 in the wavelength from $600 \mathrm{~nm}$ to $1300 \mathrm{~nm}$. The volume ratio of glass beads in epoxy is controlled by controlling the mass of the beads in the epoxy. The more the volume ratio of the glass beads in the layer, the more the absorption and scattering effect will be. Figure 3 shows the measured absorption and scattering coefficients across the volume ratio of the glass beads in transparent epoxy mimicking dermis layer at $750 \mathrm{~nm}$ wavelength. With $50 \%-60 \%$ beads volume ratio in epoxy, the absorption coefficient and scattering coefficient of the mix material are close to those of epidermis and dermis. Besides adjusting the optical properties, the beads can act as point reflection sources due to the refractive index contrast in the bottom layer while scanning the optical phantom by using RCM, which may be used to measure point spread function (PSF) for image correction. 


Refractive index around $1.52-1.54$
Thickness $-15 \mu \mathrm{m}$
Refractive index around $1.34-1.36$
Thickness $-0.1 \mathrm{~mm}$
Refractive index around $1.41-1.43$
Thickness - 1mm
Scattering coefficient $~ 1-10 \mathrm{~mm}^{-1}$
Absorption coefficient $\sim 0.1-2 \mathrm{~mm}^{-1}$

Fig. 2. Multi-layer structure design for optical phantom

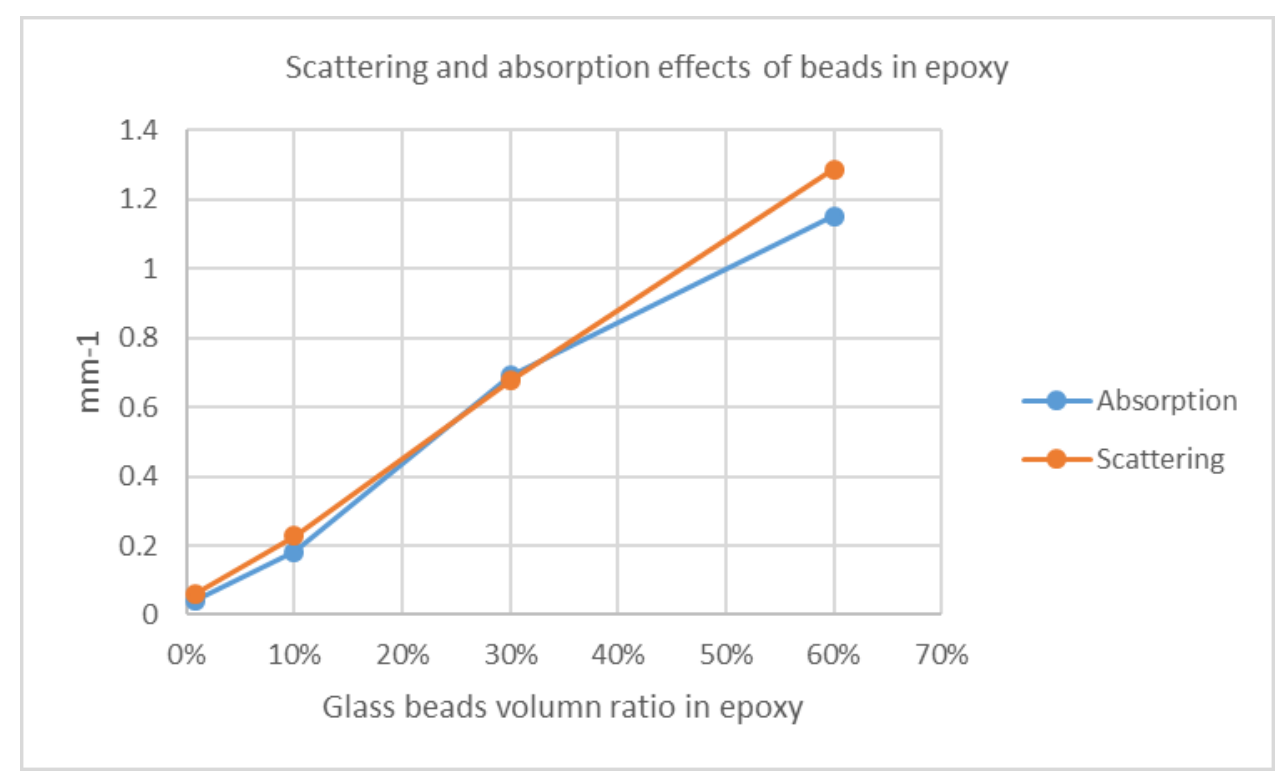

Fig. 3 Scattering and absorption effects of the beads in transparent epoxy layer

\section{Fabrication of the optical phantoms}

Commercially available transparent UV-curable resins were used to make the layers of the optical phantom. We selected resins with refractive index as close as possible to those of the correspondent skin layers. The first layer for dermis was made from transparent epoxy with refractive index of 1.46 mixed with glass beads by molding. $60 \%$ volume ratio of glass beads was used to achieve scattering and absorption coefficients similar to those of the skin. On top of the cured dermis layer, living epidermis layer was fabricated with transparent epoxy with refractive index of 1.38 without glass beads. Epoxies with low viscosity were selected for the fabrication of the layers for dermis and living epidermis to ensure good thickness control by using the designed molds. After hardening the layer for living epidermis, the fabricated dermis and living epidermis layers would be the substrate for spin coating the layer for stratum corneum. Epoxy with medium viscosity and refractive index of 1.58 was used for stratum corneum layer. The thickness of the stratum corneum layer can be controlled very well from 12 um to 30 um by varying the spin speed and time. Due to the edge effects of spin coating process, there may be coating thickness difference around the rim of the sample. Hence, once all the layers were completed, the edge of the three-layer sample was removed by laser cutting. The final sample had a size of $6 \mathrm{~cm}$ by $6 \mathrm{~cm}$. 


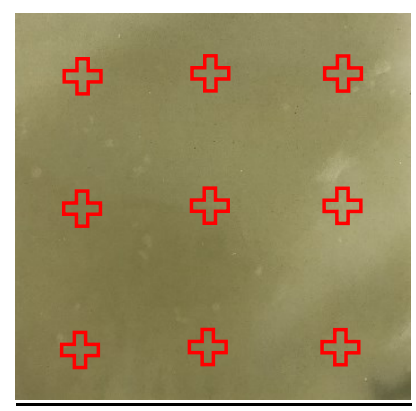

Fig. 4 Top view of the fabricated three-layer optical phantom sample.

\section{Phantom Optical Properties}

Characterization for the layers were done after each layer was fabricated. The refractive index of each layer was measured at the wavelength of $589 \mathrm{~nm}$ under room temperature after curing. The thicknesses of the transparent layer was measured across the sample area with white light interferometer, while the layers with glass beads was measured with digital micrometer at 9 locations (marked in Fig. 4) across the sample. The measurement results are listed in Table 1.

Table 1: Parameters of the optical phantom sample

\begin{tabular}{|l|l|l|l|l|l|l|}
\hline Mimic layer & $\begin{array}{l}\text { Refractive index of } \\
\text { the layer @ } \\
589 \mathrm{~nm} 20-23 C^{\circ}\end{array}$ & $\begin{array}{l}\text { With 60\% } \\
\text { volume } \\
\text { ratio } \\
\text { beads }\end{array}$ & $\begin{array}{l}\text { Designed } \\
\text { thickness of } \\
\text { the layer }\end{array}$ & $\begin{array}{l}\text { Measured } \\
\text { thickness of } \\
\text { the layer }\end{array}$ & $\begin{array}{l}\text { Scattering } \\
\text { coefficient } \\
\text { (mm-1) }\end{array}$ & $\begin{array}{l}\text { Absorption } \\
\text { coefficient } \\
\text { (mm-1) }\end{array}$ \\
\hline $\begin{array}{l}\text { Stratum } \\
\text { corneum }\end{array}$ & 1.58 & No & $15 \mathrm{um}$ & $14-16 \mathrm{um}$ & - & - \\
\hline $\begin{array}{l}\text { Living } \\
\text { epidermis }\end{array}$ & 1.38 & No & $100 \mathrm{um}$ & $90-100 \mathrm{um}$ & - & - \\
\hline Dermis & 1.46 & Yes & $1 \mathrm{~mm}$ & $1-1.07 \mathrm{~mm}$ & 1.3 & 1.2 \\
\hline
\end{tabular}

The scattering and absorption coefficients (Fig. 5) were characterized for the layer mimic dermis. The sample's scattering and absorption coefficients have the same trend across the wavelength and they are in the similar data range as those of the skin.

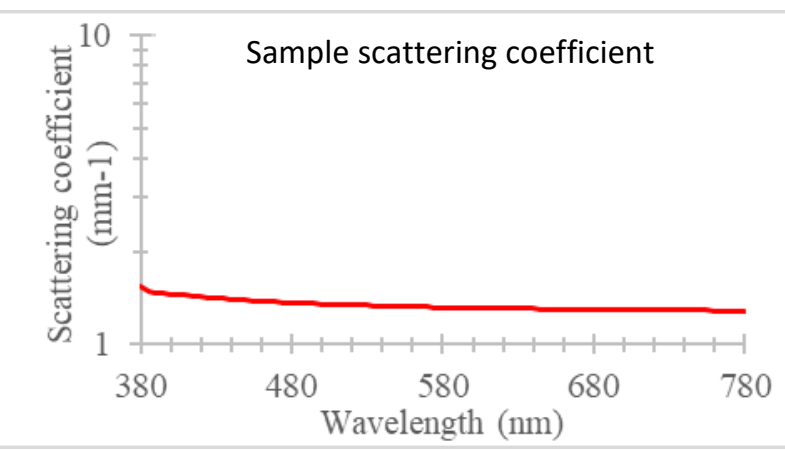

(a)

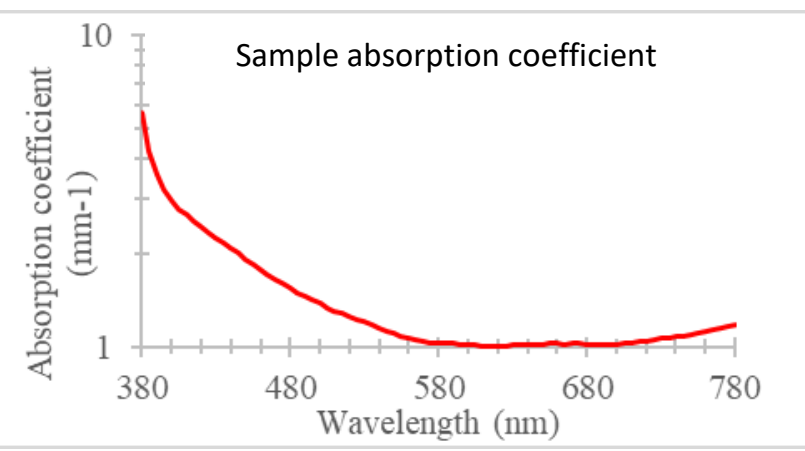

(b)

Fig. 5 The scattering (a) and absorption (b) coefficients of the sample of $1 \mathrm{~mm}$ thick cured epoxy resin with $60 \%$ of glass beads. 


\section{Phantom Optical Application in Confocal Microscopy}

Confocal acquisitions of the optical phantom is done by using a near-infra-red reflectance confocal laser scanning microscope (Vivascope 1500; Lucid Inc, Rochester, NY, USA), in the way similar to [11]. Each image corresponds to a horizontal section with a $500 \times 500 \mu \mathrm{m}$ field of view and a resolution of $1000 \times 1000$ pixels. Three vertical sequences of images, providing a three-dimensional view of a selected area, are acquired from the phantom surface to $250 \mu \mathrm{m}$ with a step of $3 \mu \mathrm{m}$.

The contrast on RCM images relies on the difference of refractivity of the tissue which depends on chemical and molecular structures. Due to these variations, only a certain portion of light is reflected. Structures with a higher refractive index, such as melanin and keratin, appears bright on RCM images.

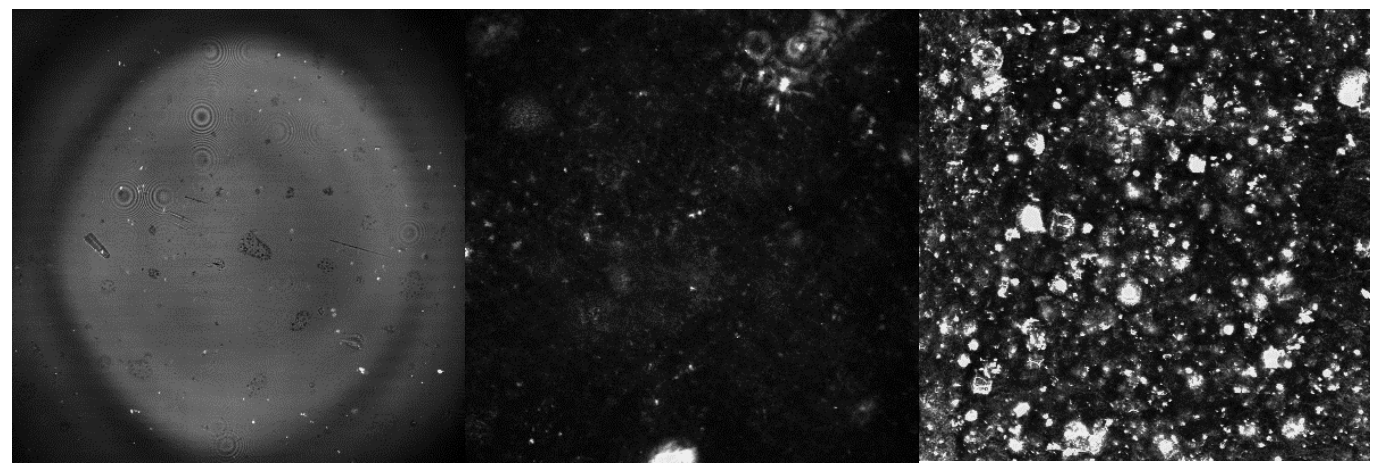

(a) Layer 3 - Stratum corneum mimic layer (b) Layer 2 - Living epidermis mimic layer (c) Layer 1 - dermis mimic layer

Fig. 6. Examples of confocal acquisitions of the optical phantom.

We aim to assess the structural properties of the optical phantom using RCM. Examples of acquisitions of images from this phantom at different depths are presented in Fig. 6 . These acquisitions allow measurement of the thickness of each layer. The mean thickness of each layer for the three acquisitions is presented in Table 2. The mean thickness of the layer 1 , corresponding to the dermis, cannot be measured as its thickness surpasses the microscope maximal imaging depth. The resulting thicknesses are overall in agreement with the phantom design and the skin structural properties. The observed variability in the measured thickness can be due to the detection of the phantom surface on RCM images which is difficult to perform by visual means. The layer 1 shows high contrast, as expected, as it is the only one containing microstructures with high refractivity indexes.

Table 2: Mean thickness of each phantom layer measured by in vivo confocal microscopy.

\begin{tabular}{|c|c|c|}
\hline Mimic layer & $\begin{array}{l}\text { Mean thickness measured using } \\
\operatorname{RCM}(\mu \mathrm{m})\end{array}$ & $\begin{array}{l}\text { Variability of the three acquisitions } \\
- \text { Standard deviation }(\mu \mathrm{m})\end{array}$ \\
\hline Stratum corneum & 13.2 & 1.7 \\
\hline Living epidermis & 94.5 & 5.3 \\
\hline
\end{tabular}

During the acquisition, confocal microscopy images are corrupted by some Poisson noise [12]. Unlike the Gaussian noise, the Poisson noise is dependent on signal, making it difficult to separate from the signal itself. The Poisson noise refers to the inherent natural variation of the incident photon flux. The images are also corrupted by spherical aberrations due to the acquisition process. To improve the quality of the images, M. Laasmaa et al. used methods of image deconvolution [13]. Even if one resorts to blind deconvolution schemes, a priori knowledge related to the Point Spread Function (PSF) is desired. The point spread function describes the response of an imaging system to a point source or point object. This knowledge can be acquired either by studying PSF theoretical properties or experimental data. Experimental PSFs may be measured using calibration beads or directly from the image by 
extracting small point-like objects [14]. Such PSFs can be used for instance to validate theoretical parametric PSF model or to assess the aberration of point spread function in given imaging systems.

Our multi-layer optical phantom contains spherical beads, which appears as high contrasted on confocal images, could be used to determine the PSF on a specific confocal microscope. One goal related to confocal macroscopy images would be to assess experimentally the PSF depth variation by imaging sample with point sources (beads) placed at different depths.

\section{Conclusion}

Optical phantoms are very useful for applications that require a very accurate optical model for human skins evaluation and they can also be helpful in clinical settings for quality control.

A three-layered scattering agent-embedded optical phantom mimicking the layer structures and optical property of the human skin was developed successfully. The visual appearance of the skin phantom under an in vivo confocal microscope shows characteristics that are in correspondence with skin. In particular, the signal attenuation in dermis, in relation with the thickness and the refractive index of the upper layers shows the same profile than the one observed in the real dermis.

Besides, the developed phantom could be used to determine the PSF for a specific confocal microscope, which could help enhance the imaging of the microscope. Despite the beads do not have the same shape than dermal fibers, they allow to have nice contrasted images that can be used for image deconvolution. Possible improvement for the optical phantom could be to have a phantom with beads in the epidermis too in order to mimic the keratinocytes cells organization.

\section{References:}

1. Vrushali R. Korde et al, Using Optical Coherence Tomography to Evaluate Skin Sun Damage and Precancer, Lasers Surg Med. 2007 Oct; 39(9): 687-695.

2. Francine Celise Siqueira et al, Pilot-study of photodamaged skin and melasma using reflectance confocal microscopy, Surg Cosmet Dermatol. Rio de Janeiro v.10 n.2 abr-jun. 2018

3. Biomimic optical phantoms, www.ino.ca/Biomimic-Optical-Phantom

4. Guy Lamouche et al, Review of tissue simulating phantoms with controllable optical, mechanical and structural properties for use in optical coherence tomography, Biomed Opt Express.; 3(6): 1381-1398. 2012

5. US patent 6224969 B1, “Optical phantom suitable for stimulating the optical properties of biological material and a method of producing said phantom", 2001.

6. US patent 20170122915 A1, "PVCP phantoms and their use", 2017.

7. Anatomy Atlases: Atlas of Microscopic Anatomy: Section 7: Integument. http://www.anatomyatlases.org/MicroscopicAnatomy/Section07/Section07.shtml.

8. H. Ding et al., "Refractive indices of human skin tissues at eight wavelengths and estimated dispersion relations between 300 and 1600 nm", Phys. Med. Biol. 51(6), 1479, 2006.

9. G. J. Tearney et al., "Determination of the refractive-index of highly scattering human tissue by optical coherence tomography," Opt. Lett. 20(21), 2258-2260, 1995.

10. Tom Lister, et al., “Optical properties of human skin”, J. of biomedical optics 17 (9), 2012

11. Milind Rajadhyaksha et al. "In vivo confocal scanning laser microscopy of human skin: melanin provides strong contrast", Journal of Investigative Dermatology 104.6, pp. 946-952, 1995.

12. Kervrann, C., Trubuil, A., "An adaptive window approach for poisson noise reduction and structure preserving in confocal microscopy", Proc. of ISBI, pp. 788-791, 2004.

13. M. Laasmaa, M. Vendelin, and P. Peterson, "Application of regularized Richardson-Lucy algorithm for deconvolution of confocal microscopy images", J. of Microscopy, Vol. 243, Issue 2, pp. 124-140, 2011.

14. Von Tiedemann M, Fridberger A, Ulfendahl M, Tomo I, Boutet de Monvel J., "Image adaptive point-spread function estimation and deconvolution for in vivo confocal microscopy", Microscopy Research \& Technique, Vol. 69, Issue 1, pp. 10-20, 2006. 